\title{
Peter Dews*
}

\section{Theoriekonstruktion und existenzielle Beschreibung in Schellings Freiheitsschrift}

DOI 10.1515/dzph-2017-0016

\begin{abstract}
Despite considerable recent attention, important features of Schelling's famous work, the 1809 treatise On the Essence of Human Freedom, remain underexplored. One of these is the methodological dualism which Schelling advocates at the very start of the text. Schelling aims to weld together into a coherent position a first-person phenomenology of freedom and an explanation achieved by locating freedom within a conceptual system articulating the basic structure of the world. Most interpretations of the Freiheitsschrift, however, concentrate on only one of these approaches, thus foreshortening their understanding of Schelling's enterprise. The article explores this tendency towards one-sidedness by considering two sophisticated recent interpretations of the work which take opposite tacks. One, by Markus Gabriel, focuses on the distinctive, self-reflexive metaphysics which Schelling proposes, while the other, by Sebastian Gardner, claims that Schelling's ontology is extrapolated entirely from his account of our moral consciousness, a procedure pioneered by Kant. The article argues that neither of these interpretations can do full justice to Schelling's project. Furthermore, although the Freiheitsschrift is not entirely successful and hence points towards later developments in Schelling's thinking, its treatment of freedom is superior to the 'soft naturalism' pioneered by Peter Strawson, and currently influential across various philosophical traditions.
\end{abstract}

Keywords: Schelling, freedom, evil, soft naturalism, Markus Gabriel, Sebastian Gardner, Peter F. Strawson

\section{Einleitung}

Schellings Abhandlung Über das Wesen der menschlichen Freiheit - die Freiheitsschrift aus dem Jahre 1809 - beginnt mit der kurzen Darstellung zweier möglicher methodologischer Zugänge, die denen offenstehen, die zu dieser The-

*Kontakt: Peter Dews, University of Essex, School of Philosophy and Art History, Wivenhoe Park, Colchester CO4 3SQ, Vereinigtes Königreich; peted@essex.ac.uk 
matik arbeiten. ${ }^{1}$ Wir können versuchen, dem Begriff der Freiheit aus der Perspektive der ersten Person Gehalt zu verleihen, indem wir introspektiv unser tief eingeprägtes Gefühl der eigenen Freiheit als selbstbewusster Akteure ans Licht zu bringen - ein Gefühl, das, wie Schelling betont, dem Ausdruck durch Worte widerstrebt. ${ }^{2}$ Alternativ können wir einen objektiven und konstruktiven Zugang wählen und uns mit der Aufgabe beschäftigen, den Freiheitsbegriff mit anderen Begriffen, mit „dem Ganzen einer wissenschaftlichen Weltansicht“3 zu verknüpfen. Eine solche Verbindung sei unerlässlich, betont Schelling, weil kein Begriff einzeln bestimmt werden könne und nur die „Nachweisung“ seines Zusammenhangs mit dem Ganzen einer Weltansicht ihm die „letzte wissenschaftliche Vollendung“ gebe. ${ }^{4}$ Wie diese Formulierung verdeutlicht, betrachtet Schelling die beiden methodologischen Optionen nicht als einander ausschließend. Er ist hingegen der Auffassung, dass die Erkundung verlangt, beiden Wegen zu folgen, und dass letztlich beide Seiten der Untersuchung ,in eins“ zusammenfallen. Sobald er aber diese Vorschläge unterbreitet hat, bemerkt er schon einen möglichen Einwand, der sich ihnen gegenüber erheben ließe: Einer seit Langem bestehenden Auffassung zufolge passe der Begriff der Freiheit mit dem des Systems einfach nicht zusammen und eine jede auf „Einheit und Ganzheit“ Anspruch erhebende Philosophie müsse unweigerlich die Möglichkeit von Freiheit leugnen. ${ }^{5}$ Dennoch hänge „individuelle Freiheit“, deren Spürbarkeit er gerade beschrieben hatte, offensichtlich ,auf irgendeine Weise mit dem Weltganzen (gleichviel ob es realistisch oder idealistisch gedacht werde)“ zusammen - womit de facto die Inkompatibilitätsbehauptung nicht zutreffen könne. ${ }^{6}$

1 Mein Dank gilt Hauke Brunkhorst, Sebastian Gardner, Wayne Martin sowie zwei unbekannten Gutachtern des British Journal for the History of Philosophy für ihre Kommentare zu einem früheren Entwurf dieser Abhandlung.

2 Schelling gebraucht das Wort „Gefühl“ nicht, um auf einen psychologischen Vorgang oder ein psychologisches Ereignis, sondern um auf eine grundlegende - vielleicht die grundlegende - Form des Selbstbewusstseins zu verweisen. Das fragliche Gefühl ist, so bekundet er, „die Tatsache der Freiheit“ (SW 1/7, 336). Offensichtlich gehören Tatsachen nicht zu jener Art von Dingen, über die man normalerweise sagt, dass sie sich „fühlen“ lassen. In einem vergleichbaren Zusammenhang, dem wir uns später zuwenden werden, bezieht sich Peter Strawson auf „dieses Gefühl des Handelns oder der Freiheit oder der Verantwortlichkeit, die wir in uns fühlen und die wir auch anderen zuschreiben“ (Strawson 1987, 41, Übers. geändert). - Schelling wird hier, wenn nicht anders angegeben, nach den Sämmtlichen Werken (Stuttgart u. Augsburg 1856 ff.) unter dem Sigel SW zitiert.

3 SW 1/7, 336.

4 Ebd.

5 Ebd.

6 Ebd. 
Schellings Abhandlung erstrebt folglich einen neuen Systembegriff, der der Freiheit Rechnung tragen kann. ${ }^{7}$

Es zeigt sich, dass eines der charakteristischen Merkmale von Schellings Ansatz darin besteht, dass sich der metaphysische Konflikt, mit dem er sich beschäftigt - zwischen der mit der Freiheit verbundenen offenen Zukunft einerseits und der Notwendigkeit, durch die sich die Welt, soll sie systematisch begriffen werden, auszeichnen muss, andererseits - auf der Ebene der Methode wiederholt. Das Problem, Freiheit innerhalb der Weltstruktur unterzubringen, findet seinen spiegelbildlichen Ausdruck in dem Problem, die Informationen, die wir aus unserer unmittelbaren, aber flüchtigen Wahrnehmung unserer selbst als freier Akteure gewinnen (Schelling behauptet, das Freiheitsgefühl sei ,unmittelbar [...] einem jeden eingeprägt" ${ }^{\text {(8) }}$, mit den Ergebnissen einer Analyse des Freiheitsbegriffs in dessen Zusammenhang mit anderen grundlegenden Begriffen zusammenzuführen. Diese innere Dualität im Denken Schellings wird durch den eingangs seiner Abhandlung formulierten Anspruch betont, dass „ohne den Widerspruch von Nothwendigkeit und Freiheit [...] nicht Philosophie allein, sondern jedes höhere Wollen des Geistes in den Tod vers[änke]“. ${ }^{9}$ Hier scheint er zu behaupten, dass die Doppelperspektive - und der Konflikt, den sie erzeugt für unser geistiges und moralisches Leben wesentlich ist und dieses in Bewegung hält. ${ }^{10}$ Es ist eines der Verdienste von Heideggers Vorlesungsreihe zur Freiheitsschrift 1936, diese Folgerung aus Schellings Grundkonzeption in den Vordergrund gerückt $\mathrm{zu}$ haben:

Die Frage nach dem System der Freiheit ist nicht nur ein „Gegenstand“ der Philosophie, sie ist auch nicht nur der eigentliche und umfassende Gegenstand, sondern sie ist zuvor und

7 Der Systembegriff, der im Deutschen Idealismus am Werke ist, betrachtet die Struktur der Wirklichkeit als eine, die logisch zwingend aus einem einzigen, höchsten Prinzip folgt (einige der Gründe für das Engagement für eine solche Auffassung werden weiter unten zum Vorschein kommen). Dies führt zu Problemen, die jenen gleichen, der der szientifische Determinismus aufwirft. Die Rolle, die F. H. Jacobi (1743-1819) für den Anstoß der intensiven nachkantischen Diskussion um System und Freiheit spielte, ist in der einschlägigen englischsprachigen Literatur in Folge der bahnbrechenden Darstellung in Beiser (1987) - mittlerweile Gemeingut.

8 SW 1/7, 336.

9 Ebd., 338.

10 In letzter Zeit steht Schellings Freiheitsschrift, des zweihundertjährigen Jubiläums (im Jahre 2009) wegen, im Mittelpunkt vieler Diskussionen, einschließlich mindestens dreier Aufsatzsammlungen, die Konferenzbeiträgen entstammen (vgl. Roux 2010, Paetzold/Schneider 2010 sowie Ferrer/Pedro 2012). Soweit ich allerdings sehen kann, finden jedoch der methodologische Dualismus, den Schelling eingangs der Abhandlung vertritt, und dessen Konsequenzen kaum Beachtung. 
im Grunde und zuerst der Zustand der Philosophie, der offene Gegensatz, in dem sie steht und den sie immer wieder zustand bringt. ${ }^{11}$

Heidegger meint hiermit, dass unsere intuitive Auffassung von Freiheit als eines „grundunbedürftigen Anfang[s]“ zwangsläufig mit der Vorstellung eines Systems als eines „geschlossene[n] Begründungszusammenhangs“ konfligiert. ${ }^{12}$ Die Philosophie muss mit der Tatsache ringen, dass die Spontaneität der Freiheit - ohne die es keine philosophische Tätigkeit geben könnte - mit ihrem eigenen grundlegenden Streben nach explanativer Schließung unvereinbar zu sein scheint. Das „System der Freiheit“, das Heidegger erwähnt - und damit lässt er eine Schlüsselidee des frühen postkantischen Idealismus anklingen -, beinhaltet aber mehr als nur eine wie auch immer erlangte Versöhnung von Freiheit und umfassender Erklärung. Die grundlegende Einsicht besteht darin, dass Freiheit dem System nicht einfach als nachträglicher Gedanke sozusagen eingeführt oder eingeimpft werden kann - denn in diesem Fall risse die Erklärungskette. Vielmehr muss das „System der Freiheit“ ein Gedankengebäude sein, das auf Freiheit gründet. Deshalb behauptet Schelling zu Beginn seiner Abhandlung, dass der Begriff der Freiheit, wenn er überhaupt Realität besitzen soll, „kein bloß untergeordneter oder Nebenbegriff“ sein könne, sondern „einer der herrschenden Mittelpunkte des Systems seyn muß“" ${ }^{13}$ Wenn allerdings Freiheit einer der zentralen Punkte der Organisation des Systems sein soll, wie ließe sie sich dann durch ihre systeminternen Verknüpfungen definieren? Wie könnte sie zugleich bedingt und unbedingt sein? Im Folgenden beabsichtige ich, diesen Fragestellungen durch die Prüfung zweier jüngst unternommener anspruchsvoller Versuche nachzugehen, die Argumentation, den philosophischen Stellenwert und die Erklärungskraft von Schellings Freiheitsschrift zu enträtseln. Bezeichnenderweise ist eine der beiden - die von Markus Gabriel - eher spekulativ oder theoretisch, die andere aber - die von Sebastian Gardner - eher praktisch oder existenziell orientiert.

11 Heidegger (1971), 69, Hervorh. im Orig.

12 Ebd., 75.

13 SW 1/7, 336. 


\section{Gabriels Interpretation der Freiheitsschrift}

In seiner kurzen Monographie Das Absolute und die Welt in Schellings Freiheitsschrift behauptet Markus Gabriel, dass Schellings Abhandlung eine charakteristische Art von Metaphysik darstelle - angenommen, die Metaphysik sei ein Versuch, „die Welt als Welt zu denken“14, im Unterschied zur Erklärung der Natur und der Existenz einzelner Entitäten oder Vorgänge in der Welt. Wie seine Zeitgenossen im Deutschen Idealismus ist sich Schelling bewusst, dass jede umfassende Darstellung der Natur der Realität reflexiv werden muss: Sie hat die Existenz solcher Darstellungen selbst einzubeziehen - und deren mögliche Geltung zu berücksichtigen -, wie auch die Tätigkeiten der Art von Akteuren unterzubringen, die solche Darstellungen erzeugen können. Die Realität ist eine solche, die metaphysische Darstellungen ihrer selbst generiert, oder zumindest ihre Generierung ermöglicht. Jede Theorie der Realität, die dieses Erfordernis nicht beachtet und unbekümmert intentione recta vor sich geht, nennt Gabriel „dogmatisch“. Im Unterschied dazu bezeichnet er Schellings Art von Metaphysik als „kritisch“. Eine kritische Metaphysik arbeitet nicht mit der stillschweigenden Annahme eines unthematisierten und damit implizit „weltlosen“ Subjekts metaphysischen Denkens - wie dies oft bei philosophischen Theorien der Fall ist, die in der Tat einem solchen Subjekt keinen Platz einräumen können. Hingegen konkretisiert sich ein kritisches Projekt à la Schelling mit der Überlegung, dass

die Welt als Welt nur dadurch ist, daß sie in eine transzendierende Bewegung hineingestellt ist, die ausschließlich von Weltwesen vollzogen werden kann. ${ }^{15}$

Zwei bedeutende Ideen sind in diesem Zitat verdichtet. Die eine ist die, dass die Welt als Welt nur kontrastiv in den Blick kommen kann: „Denn auch die Welt ist nur dadurch etwas, daß sie sich von anderem unterscheidet, das sie nicht ist.“16 Folglich meint die Formulierung „transzendierende Bewegung“ ein Streben, das über die Welt hinaus auf das zielen muss, das, was auch immer es sei, ihr negatives Gegenüber konstituiert. Gabriel fährt fort und behauptet, dass Schelling zufolge die Welt durch diese transzendierende Bewegung ihre Artikulation erlangt. Was immer es ist, das artikuliert werden mag, nennt Schelling „Wesen, sofern es bloß Grund von Existenz ist“, während er den Prozess der Artikulation „Wesen, sofern es existiert“, nennt. ${ }^{17}$ Der Grund kann seine Artikulation nicht

14 Gabriel (2006), 7.

15 Ebd., 10.

16 Ebd., 12.

17 SW 1/7, 357. 
bestimmen, denn dann wäre Letztere einfach nur eine Wirkung des Grundes, nicht aber das Ergebnis von dessen Transzendenz: Das Bewusstsein einer differenzierten Welt erfordert die freie Bewegung der Überschreitung. ${ }^{18}$ Dementsprechend kann das Existierende den Grund nicht völlig aufnehmen, denn dann gäbe es nichts, dessen Artikulation es wäre.

Betrachten wir nun, worauf die Bewegung der Transzendenz gerichtet ist. Es kann dies kein Element der Welt sein, denn in diesem Fall wäre die Transzendenz keine von der Welt. Es kann aber auch kein Element derselben Art wie die Welt sein - selbst wenn es sich von ihr angeblich unterschiede. In diesem Fall gehörte es nämlich immer noch zum selben logischen Bereich wie die Welt und würde somit zurück in ihren Orbit gezogen, statt sich ganz jenseits dessen zu befinden. Falls die transzendierende Bewegung der Artikulation einen (um hier eine bekannte Formulierung zu benutzen) allumfassenden „Raum der Gründe“ generiert, dann kann, worauf sich die Bewegung transzendierend richtet, kein „Grund“ sein. Schelling nennt es deshalb den „Ungrund“19. Von ihm kann man nicht sagen, dass er existiere (weil Schelling zufolge alles, was existiert, eine Kombination von Grund und Existierendem aufweist). Dennoch können wir ihn, so behauptet Gabriel, als das „Absolute“ betrachten, das von uns benötigt wird, damit die Welt als Welt erscheint. ${ }^{20}$

Vermutlich, um den Anschluss an vertrauteres philosophisches Vokabular zu finden, nennt Gabriel Schellings Grund und Existierendes „Substanz“ und „Struktur“ und behauptet, dass diese beiden grundlegenden Seinsdimensionen in einer instabilen, antagonistischen Wechselbeziehung stünden. Die Erklärung dafür liegt im zweiten Hauptgedanken des Zitats. Dieser besteht darin, dass die transzendierende Bewegung durch ein Wesen innerhalb der Welt ausgeführt werden muss (denn andere gibt es nicht) - was dieses Wesen in eine zweideutige Lage versetzt. Wir sind „Weltwesen“, weil wir in der Welt stehen, jedoch können wir auch aus ihr herausstehen und den (konstrastiv bedingten) Gedanken ihrer bloßen Existenz erwägen. Wir können uns selbstbewusst vom Grund scheiden. Diese Befähigung sollte uns jedoch nicht zu dem Gedanken verleiten, wir wären vom Prinzip her - ontologisch gesehen - vom Grund losgelöst: denn, so Gabriel, „[o]hne den Boden des Gegebenen, der Welt, stünden wir [...] auch nicht aus der

18 In Reaktion auf Missverständnisse seines Textes hat Schelling betont, er sei nicht seine Absicht gewesen, dass der Terminus „Grund“ als Ursache verstanden werde, sondern dass er als „Fundament, Unterlage, Grundlage, Basis“ zu verstehen sei; vgl. Brief an Georgii von 18.7.1810, in: Schelling (1870), 221.

19 SW 1/7, 407.

20 Vgl. Gabriel (2006), 37-46. 
Welt heraus“. ${ }^{21}$ Seiner Darstellung zufolge führt diese ambivalente Stellung zur Angst, die wir zu überwinden suchen, indem wir die Welt - und d. h. deren Kontingenz - beherrschen. Die Welt erscheint als kontingent, weil im Unterschied zum „Absoluten“ anderer Philosophen Schellings Ungrund per definitionem die Existenz der Welt nicht erklärt. Die Kontingenz der Welt wiederum greift auf uns Menschen über: Sie verletzt den Status, über den wir anscheinend durch unsere Fähigkeit verfügen, die Welt als ein Ganzes ins Auge zu fassen. Sie trennt uns von uns selbst und bewirkt den fundamentalen Drang menschlicher Existenz, den Gabriel „Kontingenzbewältigung“ nennt. ${ }^{22}$

Gabriel stimmte wohl zu, dass sich seine Lesart der Freiheitsschrift am besten als eine Übung in „rationaler Nachkonstruktion“ verteidigen lässt. Wie in allen solchen Übungen ist aber auch hier Umsicht gefordert, um zu verhindern, dass die Imperative, die man auf den Terminus „rational“ zurückzuführen meint, den Sinn des Originaltexts entstellen. Im Falle der Monographie Gabriels liegt, so möchte ich nun geltend machen, eine solche fehlerhafte Darstellung vor. Gewiss achtet Gabriel den idealistischen Anspruch, dass man die menschliche Freiheit, soll sie ernst genommen werden, in die Wesensdynamik des Systems einbauen muss - man muss also sozusagen dem Faden der Freiheit bis in die Tiefe folgen. So verstanden sind seine exegetischen Bestrebungen lehrreich. Weil er aber die Konsequenzen der dualen methodologischen Perspektive, die Schelling zu Beginn seiner Abhandlung umreißt, missachtet, gelangt er zu einem Freiheitsbegriff, der nicht plausibel ist.

In einer der berühmtesten Aussagen der Freiheitsschrift erklärt Schelling, der Idealismus gebe „nämlich einerseits nur den allgemeinsten, andererseits den bloß formellen Begriff der Freiheit. Der reale und lebendige Begriff aber ist, daß sie ein Vermögen des Guten und des Bösen sey. “23 Leicht ließe sich denken, eine solche Freiheit stellte für den Aufbau eines Systems hauptsächlich deshalb ein Problem dar (unmittelbar darauf folgend schreibt Schelling, diese Schwierigkeit sei „von je her“ empfunden worden und betreffe „mehr oder weniger alle“ Systeme), weil die Offenheit zur Zukunft hin, die die Ausübung des freien Willens voraussetzt, mit systemischer Geschlossenheit unvereinbar sei. Tatsächlich aber ist dieses Problem, obwohl prägnant, nicht das, worauf Schelling letztlich seine Aufmerksamkeit richtet. Genauer gesagt sieht Schelling in seiner Theorie der noumenalen Wahl des eigenen moralischen Charakters - eine Anwendung und Weiterentwicklung der Auffassung, die Kant im ersten Teil der Religion innerhalb

21 Ebd., 18.

22 Ebd.

23 SW 1/7, 352. 
der Grenzen der bloßen Vernunft vorgestellt hatte ${ }^{24}$ - eine erfolgreiche Versöhnung von Freiheit und Notwendigkeit. Obwohl unsere Handlungen zwangsläufig aus unserem empirischen moralischen Charakter fließen, ist dieser doch selbst, so argumentiert Schelling mit Kant, das Resultat unserer zeitungebundenen Wahl ihres intelligiblen Korrelats. Im Allgemeinen setzen Menschen, Schelling zufolge, irgendeinen Vorgang dieser Art voraus - obwohl natürlich üblicherweise nicht in philosophisch expliziter Form -, denn wir betrachten eine Berufung auf die spezifische moralische Beschaffenheit einer Person als keine berechtigte Entschuldigung des Unrechttuns. ${ }^{25}$ So ist für ihn die dringlichste Frage nicht mehr die nach der individuellen Freiheit gegenüber der Notwendigkeit, sondern die der Unbeständigkeit der moralischen Wahl, wobei viele Menschen das Böse wählen. Der Begriff, den, wie Schelling sagt, die unter diesem Blickwinkel betrachtete Freiheit „am auffallendsten“ trifft, sei der der „Immanenz“, womit er meint, dass durch die Einbeziehung des Bösen in die „unendliche Substanz“ oder den „Urwillen“ der „Begriff eines allervollkommensten Wesens gänzlich zerstört wird“. ${ }^{26}$

Anscheinend schwankt Schelling an dieser Stelle zwischen metaphysischen Formulierungen und einem ausdrücklich theologischen Vokabular. Für den Deutschen Idealismus ist dies allerdings geradezu typisch. Die Idealisten konnten in ihren Versuchen, die unterschiedlichen Dimensionen der menschlichen Existenz $\mathrm{zu}$ integrieren, religiöse Erfahrungen nicht weniger berücksichtigen als andere grundlegende Formen der Erfahrung. Ihre Projekte lassen sich folglich so interpretieren, dass diese - je nach der Auffassung des Interpreten - entweder eine Errettung oder eine Säkularisierung des Religiösen erfordern. Im vorliegenden Fall kann der Begriff eines „allervollkommensten Wesens“ so verstanden werden, dass er sich auf eine Welt bezieht, in der sich vom Prinzip her all ihre positiven Attribute, ohne einander zu behindern, deshalb vollständig entfalten können, weil sie aus einem unendlichen Grund fließen oder diesem Ausdruck verleihen. In der Tat ist es dies, was Schelling unter „System“ auf dieser Etappe seiner intellektuellen Entwicklung versteht. Systematizität ist daher nicht einfach ein Konstrukt, sondern die Anatomie der völlig realisierten Welt selbst, die die Philosophie $\mathrm{zu}$ rekonstruieren sucht. ${ }^{27}$ Offenkundig ist allerdings auch der ethisch-religiöse Grundton der Darstellung.

24 Kant (1977a), 688 ff.

25 SW 1/7, 383-388.

26 Ebd., 353.

27 Zu Beginn seiner Stuttgarter Privatvorlesungen aus dem Jahre 1810 behauptet Schelling, es habe „lange schon ein System gegeben, ehe der Mensch darauf gedacht hat, eines zu machen das System der Welt“; SW 1/7, 421. 
Das Problem des Bösen entsteht für Schelling, hier wiederum Kant folgend, weil das Böse auf einer Verkehrung der angemessenen Beziehung zwischen dem Allgemeinen und dem Besonderen beruht, wodurch das einzelne, empirische Selbst das Universale auszubeuten oder zu beherrschen sucht und dieses dadurch zerstört. $^{28}$ (Dies ist natürlich der Aspekt, den Gabriel aufgreift.) Ein Partikularwesen, das sich selbst den Status des Allgemeinen anmaßt, mag z. B. seine falsche Universalität durch aggressive Expansion behaupten, im Unterschied zur Eingliederung der Partikularität in die Allgemeinheit, was die sowohl ethisch als auch metaphysisch richtige Ordnung der Dinge wäre. Schelling gründet seine Argumentation aber nicht auf eine Ontologie bloßer Partikularitäten und abstrakter Universalien. Ihm zufolge wird die Beziehung zwischen Allgemeinem und Besonderem am besten durch so etwas wie das Leben eines Organismus veranschaulicht, weil dieses das Zusammenwirken der Funktionen der einzelnen Organe des Körpers durchdringt. Dennoch kann die angemessene Beziehung zwischen Allgemeinem und Besonderem immer noch entstellt werden, selbst wenn man beides als ontologische Vektoren oder Tendenzen statt als bestimmte metaphysische Stellungen versteht, wie Schelling vorschlägt. Sollten solche Verkehrungen ein dauerhaftes, nicht aufzuhebendes Merkmal der Wirklichkeit werden, gefährdete dies, so sieht dies Schelling, „Einheit und Ganzheit“ der Welt (um hier eine Formulierung eingangs der Freiheitsschrift aufzugreifen). Warum aber ist Schelling von beidem überzeugt, sowohl davon, dass die Welt eine Einheit sei, als auch davon, dass das Böse diese Einheit gefährde?

In Bezug auf den ersten Teil der Frage ist es vielleicht sinnvoll, auf einige Bemerkungen Kants über die Beschaffenheit des „natürliche[n] Gang[s] der menschlichen Vernunft“ aus der Kritik der reinen Vernunft zu verweisen:

\begin{abstract}
Zuerst überzeugt sie sich vom Dasein irgendeines notwendigen Wesens. In diesem erkennt sie eine unbedingte Existenz. Nun sucht sie den Begriff des Unabhängigen von aller Bedingung und findet ihn in dem, was selbst die zureichende Bedingung zu allem anderen ist, d. i. in demjenigen, was alle Realität enthält. Das All aber ohne Schranken ist absolute Einheit und führt den Begriff eines einigen, nämlich des höchsten Wesens bei sich und so schließt sie, daß das höchste Wesen, als Urgrund aller Dinge, schlechthin notwendigerweise da sei. ${ }^{29}$
\end{abstract}

Natürlich argumentiert Kant, dass wir uns - trotz dieser angeborenen Triebkraft der Vernunft - des Urteils, ob solch eine endgültige Quelle der Realität existiert, enthalten müssen. Für Schelling aber würde eine solche Einstellung bedeuten,

28 Für eine eingehendere Diskussion von Schellings Konzeption des Bösen im Kontext des nachkantischen Idealismus vgl. Dews (2008), Kap. 2.

29 Kant (1974), A 586-587/B 614. 
dass wir uns des Instrumentariums berauben, das wir benötigen, um uns überhaupt philosophisch zu betätigen. Sie lässt sich somit nie hinreichend begründen, ganz gleich, wie ingeniös Kant versucht, Existenzbehauptungen auf das Feld der Erscheinungen zu beschränken. Mit anderen Worten: Wird ein notwendiges Wesen als nur problematisch oder möglich beschrieben, dann widerspricht diese Charakterisierung schlichtweg der unterstellten Notwendigkeit. Jedoch besteht für Schelling das Fazit nicht darin, dass wir ein Wissen von Gott oder dem Unbedingten erlangen könnten wie ein solches von einem endlichen Ding (hier stimmt er völlig mit Kant überein). Vielmehr hält er in den ein Jahr nach Veröffentlichung der Freiheitsschrift gehaltenen Stuttgarter Privatvorlesungen fest: „Die Existenz des Unbedingten kann nicht erwiesen werden wie die des Bedingten. Das Unbedingte ist das Element, worin allein Demonstration möglich ist.“ ${ }^{\text {“30 }}$ Für ihn hat dies zur Folge, dass die letztendliche Ganzheit und Einheit der Welt als etwas, das aus einer einzelnen, absoluten Quelle stammt -, weder bewiesen noch als Vorannahme in Frage gestellt werden kann. Im Grunde genommen heißt dies, dass, falls wir nicht annähmen, dass die Realität durch ein einzelnes, letztendliches Prinzip zusammengehalten wird, wir erst gar nicht versuchten, eine Welt zu begreifen. In diesem Zusammenhang bietet die Konzeption des Bösen als einer ontologischen Verkehrung Schelling die Möglichkeit, aufzuheben, was er als die unwahrscheinlich beruhigende Konzeption vom Bösen als bloßer Privation des Guten betrachtet, ${ }^{31}$ und zugleich dem Bösen die endgültige innere Konsistenz abzusprechen. Er muss zeigen, dass die Verkehrung nicht als ein ursprüngliches und permanentes Merkmal der Realität konzipiert zu werden braucht (sondern als befristetes, allerdings nachhaltiges, konzipiert werden kann), weil andernfalls die Welt zwei miteinander unverträgliche Gründe hätte. Nicht nur wäre damit der Erklärungsbedarf schlichtweg um eine Stufe zurückgesetzt, sondern der daraus resultierende Manichäismus würde unsere sittliche Orientierung zerstören.

Im Unterschied dazu verschwindet in Gabriels Rekonstruktion der Begriff des Bösen als einer Verkehrung völlig, weil er es als die Verfassung darstellt, die einem selbstbewussten aber endlichen Wesen inhärent ist: „Frei zu sein heißt aber, böse zu sein, weil alle freien Wesen Weltwesen sind und Weltwesen aufgrund ihrer instabilen Struktur notwendig versuchen, die Welt zu beherrschen. Es gibt kein freies Individuum, das nicht radikal böse ist. “32 Wenn er aber behauptet,

30 SW $1 / 7,423$.

31 Ebd., 370-372.

32 Gabriel (2006), 32-33. 
dass die „Freiheit [...] dem Menschen nur zu[kommt], weil er radikal böse ist““33, oder dass es „zur Dynamik des Ichs“ gehöre, „böse zu sein“, dann steht dies in direktem Widerspruch zu Schellings Behauptung, Freiheit sei ein Vermögen zum Guten oder zum Bösen. Durch die Verwendung der Metaphern von Zentrum und Peripherie, die seinen Text durchziehen, vergleicht Schelling das Böse mit einem Leiden, das ein Organ befallen hat, das seine spezielle, untergeordnete Rolle im übergreifenden Funktionszusammenhang des Organismus spielen sollte, aber danach verlangt, ins Zentrum zu treten, Herrschaft zu erlangen, und dadurch den Körper als Ganzen in einen Zustand von Schmerz und Unordnung bringt. ${ }^{34}$ Es ist schwer zu verstehen, wie eine solche Krankheit - die Schelling als das „wahre Gegenbild des Bösen“35 beschreibt - als die Gestalt verstanden werden kann, die die menschliche Freiheit annimmt.

Zugegebenermaßen erklärt Schelling an einer Stelle der Freiheitsschrift, der „Eigendünkel des Menschen sträubt sich gegen diesen Ursprung aus dem Grunde“. ${ }^{36}$ Scheinbar wird damit Gabriels Behauptung bestätigt, dass wir unweigerlich von einem Projekt der Kontingenzbewältigung in Anspruch genommen sind. Schelling aber behauptet mit Nachdruck, es sei „nicht die erregte Selbstheit an sich [...] das Böse, sondern nur sofern sie sich gänzlich von ihrem Gegensatz, dem Licht oder dem Universalwillen, losgerissen hat". ${ }^{37}$ Wie wir gesehen haben, sind Grund und Existierendes nach Schellings Konzeption nicht separate, starre Seinsschichten, sondern lassen sich besser als Drang oder Streben des Seins zur Vereinzelung oder zur Verallgemeinerung hin beschreiben. Er hebt dieses Merkmal auch dadurch hervor, dass er auf sie anthropomorphisierend als „Partikularwille“ und „Universalwille“ verweist. Diese Willen können als „protoontologisch“ bezeichnet werden, weil jedes wirklich existierende Ding aus einer spezifischen Spannung und einem spezifischen Gleichgewicht von Grund und Existierendem besteht. In einer Hinsicht widerstreiten beide einander: Der Partikularwille strebt danach, seine Partikularität gegenüber dem Universalwillen zu behaupten, während der Universalwille vor seiner Zersetzung durch den Partikularwillen zurückweicht. Zugleich aber sind beide zutiefst verflochten. Der Partikularwille oder Grund ist nicht einfach ein undurchsichtiger Prozess des Sich-Zurückziehens und der Selbstrückbildung, sondern das, was Schelling einen „Willen zur Offenbarung“ nennt, der, „damit diese sei, [...] die Eigenheit

33 Ebd., 50.

34 SW $1 / 7,366$.

35 Ebd.

36 Ebd., 360.

37 Ebd., 400. 
und den Gegensatz hervorrufen“ müsse. ${ }^{38}$ Als Partikulares kann er nicht offenbar werden, außer durch Rückgriff auf Allgemeinbegriffe. Im Gegenzug bedarf

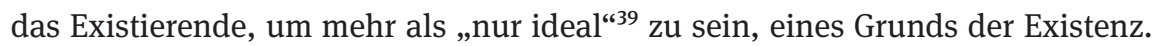
Da jede Gestalt des Willens in beide Richtungen - sowohl dem anderen entgegen als auch auf das andere $\mathrm{zu}$ - strebt, gibt es keinen Grund, warum sie nicht schließlich verschmelzen sollten. Eine solche Vereinigung beinhaltete allerdings nicht die vollständige Auflösung des Materialen im Idealen oder des Partikularen im Universalen. Schellings überzeugendstes Bild für diesen Vorgang betrifft die Art und Weise, wie ein durchsichtiger Körper, ohne aufzuhören, Materie zu sein, „Träger und gleichsam Behälter des [...] Lichts“ wird. ${ }^{40}$

Gabriel also gelingt es im Wesentlichen nicht, den zutiefst dialektischen Charakter von Schellings Konzeption zu erkennen, in der die Identität jedes Seinsvektors nicht allein gewissermaßen abhängig von der Beziehung auf sein Gegenüber, sondern durch dieses intern gespalten ist. Für ihn ist die Beziehung zwischen Existierendem und Grund eine einseitige und hauptsächlich epistemische. Der Ersatz von Schellings plastischer Konzeption einer Entwicklungsbeziehung zwischen Partikular- und Universalwillen, dessen Interaktion die Formen der natürlichen Welt entstehen lässt, durch die eher schwergängigen Begriffe „Substanz“ und „Struktur“ ist in dieser Hinsicht bezeichnend. So schreibt er: „Was beobachtet wird, ist letztlich die Substanz der Welt. Wie sie beobachtet wird, ist ihre Struktur.“ “41 Diese Lesart, derzufolge der Substanz die Struktur durch den Wissenden offenbar auferlegt wird, setzt sich einfach über Schellings Absicht hinweg, eine Darstellung der Beziehung von Partikularem und Universalem zu liefern, die sowohl sittlich als auch metaphysisch tragfähig ist. ${ }^{42}$ Gabriel gelingt es, anders gesagt, nicht, zu erläutern, wie Struktur die Struktur der Substanz in dem Sinne dem des Genitivus subiectivus - sein kann, den die Freiheitsschrift eindeutig meint. Sein Versuch, dieses Problem durch Anwendung des Ausdrucks „Struktur“ auf die Beziehung zwischen Substanz und Struktur zu erledigen, macht das Problem nur noch schlimmer: Entweder verletzt er damit seine eigene Definition

38 Ebd., 375.

39 Ebd., 410.

40 Ebd., 364.

41 Gabriel (2006), 42, Hervorh. getilgt.

42 An einer Stelle diskutiert Gabriel eigentlich die Tatsache, dass „der Grund“ „zum Verstand“ „tendiert“ (ebd., 22), aber im Grundtenor seiner Auffassung ist die Beziehung von Struktur und Substanz eine aufgezwungene. Obwohl er am Schluss seiner Monographie einräumt, Schellings Ziel sei die Übereinstimmung von Substanz und Struktur (oder von „Inhalt und Form“), hat seine Interpretation dies undenkbar gemacht und nötigt ihn zur Berufung auf einen „göttlichen Akt der Gnade“ - ein Gedanke, der sich in Schellings Abhandlung nicht findet (vgl. ebd., 51). 
von Struktur oder er produziert damit einen Regress (vgl. z. B.: „Indem Substanz und Struktur der Welt sich wechselseitig voraussetzen und bedingen, bilden sie eine symmetrische Struktur ${ }^{\star 43}$ ).

Demgemäß reduziert Gabriel die Freiheit auf den - vornehmlich eher kognitiv als praktisch verstandenen - Drang der Transzendenz als solchen. „Das Weltwesen denkt die Welt. Das ist seine Freiheit. “44 Das Ergebnis ist eine allgemeine Darstellung der Dynamik menschlicher Existenz als durch kontingente Individuation und den Versuch charakterisiert, die Kontingenz durch Auferlegung von Struktur zu überwinden. Das Fazit bildet Gabriels Behauptung, dass das „Böse [...] das Individuum ist, das sich gegen die Welt behauptet““. ${ }^{45}$ Jedoch muss er, um eine solche Lesart vorzuschlagen, jene Textpassagen der Freiheitsschrift außer Acht lassen, in denen Schelling die Erfahrung beschreibt, durch die Wahl zwischen Gut und Böse zerrissen zu sein. Als gut gilt Schelling jene Identität des Partikularwillens mit dem Universalwillen, für die er hauptsächlich das Bild des geometrischen Verhältnisses von Zentrum und Peripherie eines Kreises anbietet. Im Fall der Menschen ist der Partikularwille selbstbewusst geworden und fürchtet deshalb den Verlust des Selbst, den sein Zusammenfallen mit dem Universalwillen zur Folge hätte - auch wenn dies sittlich erforderlich ist. Schelling schreibt dazu: „Die Angst des Lebens selbst treibt den Menschen aus dem Centrum, in das er erschaffen worden; denn dieses als das lauterste Wesen alles Willens ist für jeden besonderen Willen verzehrendes Feuer; um in ihm leben zu können, muß der Mensch aller Eigenheit absterben, weßhalb es ein fast nothwendiger Versuch ist, aus diesem in die Peripherie herauszutreten, um da eine Ruhe seiner Selbstheit zu suchen. “46 Bemerkenswerterweise nutzt Schelling den Ausdruck „ein fast nothwendiger Versuch“: Er behauptet nicht, das Böse sei unwiderstehlich; auch machte allerdings seine Definition der Freiheit als eines Vermögens zum Guten oder zum Bösen keinen Sinn, wenn er dies täte. In ähnlicher Weise beschreibt er, wie der Wille des Grundes „nothwendig gegen die Freiheit als das Übercreatürliche [reagiert] und [...] in ihr die Lust zum Creatürlichen [erweckt], wie den, welchen auf einem hohen und jähen Gipfel Schwindel erfaßt, gleichsam eine geheime Stimme zu rufen scheint, daß er herabstürze“ “. ${ }^{47}$ Obwohl diese Reaktion des Grundes auf den abgründigen Charakter der Freiheit

43 Ebd., 39.

44 Ebd., 36.

45 Ebd., 30.

46 SW $1 / 7,381$.

47 Ebd., 381. 
unausweichlich ist, behauptet Schelling nicht, wir seien gezwungen, dem Ruf der Stimme zu folgen.

Diese letzte Textpassage unterstreicht die Tatsache, dass Gabriels Interpretation zufolge die Freiheitsschrift nicht erreichen kann, was er selbst als ihre Zielsetzung angibt. Für Schelling bezeichnet Freiheit - das bestimmende Merkmal einer Entität, die fähig ist, Metaphysik zu treiben - eine Stellung über und jenseits der Kreatur (jenseits von allem mit einer gegebenen Natur). Nur von einem solchen Standpunkt aus können wir flüchtig die Existenz der Welt als ganzer in den Blick bekommen. Dies bedeutet, dass für Schelling Welttranszendenz untrennbar mit der Erfahrung verbunden ist, wählen zu müssen. Die Welt zu transzendieren heißt nämlich, keine Natur zu haben, die vorschreibt, was man tut - im Unterschied zu Entitäten, die durch ihre Rolle und Stellung in der Welt gänzlich festgestellt sind. In diesem Zusammenhang versteht Schelling die Wahl zwischen Gut und Böse als paradigmatisch, denn in unseren sittlichen Wahlentscheidungen bestimmen wir, wer wir im Grunde sind. Folglich lassen sich diese Entscheidungen nicht lediglich als Wirkungen dessen, wer wir sind, begreifen. ${ }^{48}$ So schreibt Schelling in den Abhandlungen zur Erläuterung des Idealismus in der Wissenschaftslehre aus den Jahren 1796/1797, dass wir uns „der Freiheit [...] nicht bewußt [werden] als durch Willkür, d. h. durch die freie Wahl zwischen entgegengesetzten Maximen, die sich wechselseitig ausschließen und in einem und demselben Wollen zusammen nicht bestehen können“.49 Wenn wir hingegen durch das Böse schlechthin bestimmt und wenn unsere Erkenntnisbemühungen unweigerlich nur angstgetrieben sind, was dann den Drang nach Kontingenzbewältigung entstehen lässt, wenn wir uns immer schon vom Gipfel gestürzt haben, dann sind wir nicht in der Lage, uns von der Welt zu lösen, um sie als Ganzes zu begreifen. Die Fähigkeit, die Welt in den Blick zu nehmen, setzt eine Freiheit voraus, die Gabriels Theorie der Freiheit als deckungsgleich mit dem Bösen (mit praktisch-epistemischem Egozentrismus) verneint.

Um zusammenzufassen: Gabriel kommt zu seiner Interpretation nur, indem er den methodologischen Dualismus ignoriert, den Schelling eingangs seiner Abhandlung anführt. Gewiss beabsichtigt Schelling, gemeinsam mit den anderen Vertretern des Deutschen Idealismus, eine Metaphysik zu erstellen, die der Existenz von Wesen Rechnung zu tragen vermag, die Metaphysik treiben können. Er erwägt jedoch nicht, dass die Theorie der Freiheit und ihrer Rolle in der Welt,

48 Diese Auffassung lässt sich durch die Überlegung stützen, dass uns allein Gewissensbisse die Schädigung unserer Wesensidentität spüren lassen - wie sehr wir auch andere Mängel - wie etwa an Klugheit, Stärke, Kreativität - bedauern mögen.

49 SW 1/1, 440, Hervorh. getilgt. 
die wir letztlich formulieren, die Phänomenologie der Freiheit, wie sie in unserer unmittelbaren Selbstwahrnehmung offenbar wird, nicht einfach ignorieren kann. Das wirklich grundlegende Merkmal dieser Phänomenologie aber ist die beständige Forderung, Entscheidungen treffen zu müssen. Das menschliche Leben ist, so ließe sich sagen, eine endlose Reihe kleiner und großer moralischer Notfälle. Dementsprechend lehnt Schelling die Auffassung ab, dass der Drang nach instrumenteller Beherrschung (Gabriels Kontingenzbewältigung) unser Selbstsein zwangsläufig begleitet. Wir sind nicht grundsätzlich durch einen Drang zur Herrschaft bestimmt, von dem Schellings Dialektik von Grund und Existierendem zeigt, dass er kontraproduktiv ist. Seiner Auffassung nach liegt das Grundproblem in der Vorrangigkeit des Selbstseins - sie ist die Quelle des Antagonismus, statt dessen unausweichliche Folge zu sein. Ohne eine solche Vorrangigkeit gäbe es keinen schroffen Gegensatz von Universalität und Partikularität und somit auch keinen Zwang: Die Menschen würden zweifellos viele instrumentelle Maßnahmen, um ihr Überleben wie auch ihr Wohlergehen zu sichern, ergreifen. Aber dies könnte innerhalb eines Rahmens der Gegenseitigkeit von menschlicher und natürlicher Welt erfolgen.

Gabriels Konzeption der Freiheitsschrift hauptsächlich als einer sich selbsteinschreibenden metaphysischen Theorie vereinfacht Schellings Methode und verkürzt dessen Analyse der Freiheit. Sie kann deshalb keine Antwort auf die Frage liefern, wie Theorie und existenzielle Beschreibung im Text zusammenpassen. Einer Interpretation, die die praktische Dimension und das Freiheitsbewusstsein der ersten Person betont, die aber auch Schellings metaphysische Behauptungen aus einem solchen Bewusstsein $\mathrm{zu}$ erschließen versucht, ist deshalb vielleicht eine größere Erfolgsaussicht beschieden. Dies ist Gardners Ansatz.

\section{Gardners Interpretation der Freiheitsschrift}

Statt die Freiheitsschrift als dem Erfordernis nach einer kritischen, selbstreflexiven Metaphysik geschuldet zu verstehen, unterbreitet Gardner den Interpretationsvorschlag, Schelling gelange ausgehend von einer Einstellung des praktischen Bewusstseins zu Schlussfolgerungen, die den endgültigen Charakter des Seins betreffen. Er schreibt: „Schellings Argument von der Praxis zur Theorie sollte stattdessen mit den Begriffen erfasst werden, die Paul Franks als allgemeinen Weg zum Verständnis der Entwicklung des Deutschen Idealismus vorschlug. Franks argumentiert, dass Kants Deduktion des Sittengesetzes in der zweiten Kritik, sein Begriff vom Faktum der Vernunft, eine Blaupause für den Deutschen Idealismus sei, der die Strategie übernimmt, einen ontischen Grund - im Deut- 
schen Idealismus: die Wirklichkeit des intelligiblen Grundes der Welt, das ens realissimum - aus einem epistemischen Grund, nämlich unserem Bewusstsein

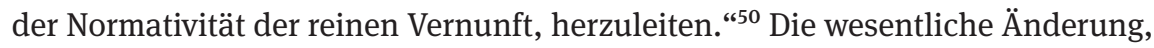
die Schelling an Kants Verfahren vornimmt, besteht Gardner zufolge darin, der Zwiespältigkeit unserer moralischen Situation das gebotene Gewicht zu verleihen: Wir sind nicht nur fähig, das Sittengesetz als normativ positive Tatsache zu respektieren, die uns die Wirklichkeit der Freiheit offenbart, wir sind auch zutiefst dafür anfällig, dem Sog des moralisch Bösen zu erliegen, wobei dieses als Verkehrung oder Negation der Normativität betrachtet wird. Während für Kant die Quelle des Bösen eine nachrangige Erwägung ist, die den Gebrauch nicht beeinträchtigt, den er vom Faktum der Vernunft (unsere Empfänglichkeit für die imperativische Kraft der reinen praktischen Vernunft) macht, sollte Schelling zufolge bei der Beschreibung unseres praktischen Bewusstseins der Reiz des Bösen gleichermaßen im Vordergrund stehen und eingehend untersucht werden. Daraus ergeben sich, so Gardners Interpretationsvorschlag, die beträchtlichen Unterschiede zwischen Schellings und Kants Metaphysik.

Gardners Interpretation enthält bedeutende Einsichten. Gewiss bestand eine der Absichten des jungen Schelling darin, Kants Konzeption des Wollens zu korrigieren, indem er dem sittlich Bösen den Stellenwert einer aktiven Gegenkraft einräumte. In seiner Metaphysik der Sitten hatte Kant den Interpretationsvorschlag unterbreitet, eine Wahl könne nur dann wirklich frei sein, wenn sie sich dem Sittengesetz unterwerfe, denn allein durch die Erfahrung, die wir mit uns als moralisch Handelnden machen, bekunde die Freiheit ihre Wirklichkeit: Es gebe keinen Grund, sich als frei zu betrachten, wenn man durch natürliches Begehren getrieben handelt. Zugleich könne aber allein die eigene Unterordnung unter das Sittengesetz kein Akt der Freiheit sein, ausgenommen, es gebe eine wirklich freie Wahl zwischen Alternativen, zwischen gut und böse. Vom praktischen Standpunkt aus gesehen lasse sich deshalb nicht verstehen, wie Moralität die Verwirklichung von Freiheit sein kann, weil das Sittengesetz keine freie Alternative zulässt, während es vom theoretischen Standpunkt aus unverständlich sei, wie aus der freien Wahl Moralverstöße resultieren können. Kant schreibt, dass, obgleich

der Mensch, als Sinnenwesen, der Erfahrung nach ein Vermögen zeigt, dem Gesetze nicht allein gemäß, sondern auch zuwider zu wählen, dadurch doch nicht seine Freiheit als intelligiblen Wesens definiert werden könne, weil Erscheinungen kein übersinnliches Objekt (dergleichen doch die freie Willkür ist) verständlich machen können, und daß die Freiheit

50 Gardner (2017), 232-233. Gardner bezieht sich auf die Darstellung der zentralen Bedeutung von Kants „Faktum der Vernunft“ für die Methodologie des Deutschen Idealismus, wie sie bei Franks (2005, Kap. 5) gegeben wird. 
nimmermehr darin gesetzt werden kann, daß das vernünftige Subjekt auch eine wider seine (gesetzgebende) Vernunft streitende Wahl treffen kann, wenn gleich die Erfahrung oft genug beweist, daß es geschieht (wovon wir doch die Möglichkeit nicht begreifen können). ${ }^{51}$

Wir haben schon bemerkt, dass Schelling in seinen früheren Kommentaren zu Fichtes Wissenschaftslehre unser Freiheitsbewusstsein als ein Bewusstsein der Wahl verstanden hatte. Kants Auffassung von Freiheit als einer uns von empirischen Regungen befreienden Autonomie - jene Konzeption, der seine Schwierigkeiten geschuldet sind - sei unangemessen, denn „wir können uns keine positiv moralische Handlung denken, ohne ihr eine positiv unmoralische entgegenzusetzen. Diese Entgegensetzung muss real sein, d. h. beide Handlungen müssen im Bewußtsein als gleich möglich vorkommen“. ${ }^{52}$ Aus seinem Gedanken zog Schelling jedoch den Schluss, dass die durch uns erfahrene Macht der Willkür lediglich die Erscheinung der absoluten Freiheit, eines „ursprünglichen Willens“ sei, „dessen Stimme nur durch das Medium der Vernunft zu uns gelangt “.${ }^{53}$ Unsere Erfahrung der positiven Sogwirkung des moralisch Bösen ist gewissermaßen eine transzendentale Bedingung der Erfahrung der Wahl, wurde aber nicht so verstanden, als entspräche ihr etwas auf der Ebene der Ontologie oder Metaphysik.

Im Jahre 1809 hält Schelling diese Lösung nicht mehr für zufriedenstellend, weil sie leugnet, dass der moralischen Wahlentscheidung letzte Wirklichkeit zukommt, und sie diese zu einer der „nothwendigen Schranken unserer Natur“54 herabsetzt. Dieser Darstellung zufolge ist in der intelligiblen Welt unsere Freiheit „nichts anderes als die absolute Bestimmung des Unbedingten durch die bloßen (Natur-)Gesetze seines Seyns“55. Schelling kommt, anders gesagt, letztlich zu dem Schluss, dass eine solche absolute noumenale Determination überhaupt keine Freiheit ist (sie ist kurz gesagt Kants „Freiheit eines Bratenwenders“), und sich mit der bloßen Erscheinung sittlicher Freiheit abzufinden, fiele hinter die systematischen Ambitionen des Idealismus zurück: nämlich eine genuine Integration der scheinbar miteinander unvereinbaren Weisen zu erlangen, auf die Menschen sich und die Welt erfahren. Somit ist eine radikal überarbeitete Ontologie gefordert, in der die Instabilität der moralischen Wahlentscheidung die prekäre Beziehung zwischen Grund und Existierendem - in die Struktur der Dinge verwoben ist (die Instabilität muss also sozusagen bis ins Fundament

51 Kant (1977b), 333, Hervorh. getilgt.

52 SW 1/1, 430, Hervorh. getilgt.

53 Ebd., 433.

54 Ebd., 439.

55 Ebd., 437, Hervorh. getilgt. 
reichen). Im Ergebnis legt Schelling in der Freiheitsschrift nicht mehr nur, um die kantsche Konzeption vom Faktum der Vernunft einfach zu ergänzen, den Akzent auf den „Hang zum Bösen“; denn er ist nicht länger davon überzeugt, dass es so etwas wie die Normativität der reinen praktischen Vernunft gibt. Die folgende Passage zeigt dies:

Wenn das Böse in einer Zwietracht der beiden Principien besteht, so kann das Gute nur in der vollkommenen Eintracht derselben bestehen, und das Band, das beide vereinigt, muss ein göttliches seyn, indem sie nicht auf bedingte, sondern auf vollkommene und unbedingte Weise eins sind. Das Verhältnis beider läßt sich daher nicht als selbstbeliebige, oder aus Selbstbestimmung hervorgegangene Sittlichkeit vorstellen. Der letzte Begriff setzte voraus, daß sie nicht an sich eins seyen; wie sollen sie aber eins werden, wenn sie es nicht sind? ${ }^{56}$

Schelling stellt klar, dass sich für ihn das sittlich Gute nicht in einem rationalen Willen findet, der unabhängig von unserem empirischen Selbstsein agiert (mit der Folge, dass die Frage, ob unsere Impulse in dieselbe Richtung oder in die entgegengesetzte Richtung drängen, für die sittliche Einschätzung des Willens ohne Belang ist). Im Gegenteil sind Grund und Existierendes, der Partikularwille und der Universalwille, in der sittlichen Person völlig miteinander verschmolzen. Wahrhaft sittlich zu sein kann nicht in rationaler Selbstbestimmung bestehen: Falls wir uns nämlich zum richtigen Tun erst bestimmen müssen, befinden wir uns bereits in einem Zustand innerer sittlicher Spaltung, und der selbstauferlegte Zwang kann die beiden Prinzipien in uns nicht zur Einheit bringen. ${ }^{57}$ Natürlich ließe sich immer einwenden, Schelling verstehe die betreffende Moralpsychologie falsch. Allerdings präsentiert er später in der Freiheitsschrift eine eingehendere Darstellung der psychologischen Folgen seiner Metaphysik. Dabei bietet seine Beschreibung zumindest eine plausible Alternative zu dem, was er als „unsere negative Moral“ bezeichnet - worin sich seine grundlegende Überzeugung niederschlägt, dass Partikularität (der „Grund“) das Universale (das „Existierende“) zu stützen und zu aktivieren vermag, aber von letzterem nie vollständig neutralisiert oder aufgenommen werden kann:

56 SW 1/7, 392.

57 Schelling nimmt in diesem Kontext Bernard Williams’ bekanntes „Ein-Gedanke-zu-viel“-Argument vorweg: „Derjenige ist nicht gewissenhaft, der sich im vorkommenden Fall noch erst das Pflichtgebot vorhalten muß, um sich durch Achtung für dasselbe zum Rechtthun zu entscheiden“ (SW 1/7, 392). Eine sorgfältige, die Zusammenhänge entfaltende Analyse der Freiheitsschrift als einer Kritik des kantischen Begriffs der Freiheit als rationaler Autonomie findet sich in Dörendahl (2011). 


\begin{abstract}
Ein Gutes ohne wirksame Selbstheit ist selbst ein unwirksames Gutes. [...] Die Leidenschaften, welchen unsere negative Moral den Krieg macht, sind Kräfte, deren jede mit der ihr entsprechenden Tugend eine gemeinsame Wurzel hat. Die Seele alles Hasses ist Liebe, und im heftigsten Zorn zeigt sich nur die im innersten Centrum angegriffene und aufgereizte Stille. Im gehörigen Maß und organischen Gleichgewicht sind sie die Stärke der Tugend selbst und ihre unmittelbaren Werkzeuge. ${ }^{58}$
\end{abstract}

Aber nicht einfach der Begriff der reinen praktischen Vernunft verfehlt die Erscheinungen unseres sittlichen Lebens. Im Wesentlichen meint Schelling, dass - wie Kant selber einräumt - eine Theorie der sittlichen Person, die sie als entzweit zwischen transzendentaler Freiheit und den Verlockungen empirischer Anreize beschreibt, die Attraktion des Bösen überhaupt nicht erklären könne. Weil Kant sich darauf festgelegt hat, was Henry Allison folgend die „Inkorporations-These“ genannt wird (die Ansicht nämlich, dass kein empirischer Anreiz uns zum Handeln zu bewegen vermag, ausgenommen, er wird in eine Maxime aufgenommen ${ }^{59}$, kann er nicht erklären, wie Menschen geneigt sein könnten, ein Grundprinzip anzunehmen, das empirischen Anreizen von vornherein eine dominierende Rolle zuweist. Genau aus diesem Grund ist Schelling bestrebt, eine neue Theorie des Wollens als „Urseyn“60 auszuarbeiten, die die Abstoßung und Anziehung seiner partikularisierenden und universalisierenden Dimensionen hervorhebt.

Diese Theorie befähigt ihn, ein Narrativ der sich entfaltenden Interaktion zwischen Grund und Existierendem zu konstruieren, die im Auftreten von Menschenwesen kulminiert - also in solchen Wesen, die zu selbstbewusster Freiheit fähig sind. Eine zeitgenössische Analogie zu diesem Schritt, die vielleicht hilft, ihn etwas verständlicher zu machen, liefert Thomas Nagels Argument hinsichtlich der Präsuppositionen des „Wertrealismus“. Ihm zufolge ist selbst der Wertreduktionismus dem Realismus mindestend in Bezug auf einige Werte verpflichtet - zum Beispiel in Bezug auf den der Wahrheit. Wenn man dies einmal zugegeben hat, müsse darüber hinaus eingeräumt werden, dass der „Wertrealismus die Tatsache verständlich machen muss, dass der biologische Entwicklungsprozess und die physikalische und chemische Geschichte, die ihm vorhergegangen sind, zu bewussten Geschöpfen geführt haben, zu den wirklichen Werten, von denen deren Leben und Erfahrungen erfüllt sind“ “. ${ }^{61}$ Den Wert als

58 SW 1/7, 400-401.

59 Der Begriff ,Inkorporationsthese (,incorporation thesis`) wurde eingeführt in Allison (1990), $5-6$.

60 Ebd., 350.

61 Nagel (2012), 117. 
kontingentes Produkt wertfreier Prozesse $\mathrm{zu}$ betrachten, implizierte jedoch, ihm die Bedeutung zu nehmen, widerspräche seiner Wesenhaftigkeit, die wir letztlich nicht anders als ernst nehmen können. Mithin sei die „teleologische Hypothese“ erforderlich, „dass diese Dinge nicht nur durch wertfreie Chemie und Physik determiniert sind, sondern auch durch etwas anderes, nämlich eine kosmische Prädisposition zur Bildung von Leben, Bewusstsein und dem Wert, der von ihnen untrennbar ist". ${ }^{62}$

Solche Schlussfolgerungen - für die Nagel Vorläufer im Deutschen Idealismus vermerkt ${ }^{63}$ - unterscheiden sich erheblich von dem durch Gardner beschriebenen Prozess. Wie wir gesehen haben, ist dessen Vermutung die, dass Schelling, indem er Kants Gedankenbewegung vom praktisch sittlichen Bewusstsein $\mathrm{zu}$ theoretischen Resultaten folgt, die Freiheit und das Böse als „letztgültige ontologische Fakten“ vorgestellt und dadurch das Verlangen nach einer weitergehenden Erläuterung gestillt habe. ${ }^{64}$ Allerdings ist Kant nur in der Lage, das sittliche Bewusstsein als ratio cognoscendi der Freiheit $\mathrm{zu}$ benutzen, weil er glaubt, dass wir uns der Moralität als einer Forderung bewusst sind, einem Prinzip universaler Gesetzgebung Folge zu leisten. Möglichst einfach ausgedrückt: Wenn das menschliche Bewusstsein einer imperativischen Form des Unbedingten begegnen kann, dann dürfen wir daraus schließen, dass es als Bedingung der Möglichkeit dieses Imperativs eine unbedingte Dimension des eigenen Seins gibt, selbst wenn alles, was wir ansonsten erfahren können, inklusive des eigenen inneren Lebens, einem strukturierten Bereich von empirischen Abhängigkeiten zugehört. Allerdings wissen wir jetzt, von Schelling belehrt, dass wir, wenn wir die Moralität so denken, nicht erklären können, wieso uns das Böse überhaupt anzieht. Schelling zufolge sollte man das, was innerhalb des Rahmens einer Dualismus-verewigenden Deontologie oder dergleichen wie Normativität aussieht, als einen grundsätzlichen ontologischen Sog ansehen, der aus der nichtversöhnten Dichotomie, die das Sein durchdringt, entsteht, und der eine entsprechende Gegenkraft hervorbringt. Um seine metaphysischen Schlüsse zu stützen, kann Schelling somit kein Bewusstsein reiner Normativität heranziehen. Wie seine Fichtekritik am Anfang der Freiheitsschrift klarmacht, ${ }^{65}$ sähe er ein solches Verfahren als methodologisch verdächtig an. Außerdem beschreibt er den Unterschied zwischen dem Grund und dem Existierenden, den er später

62 Ebd., 123.

63 Ebd., 17.

64 Gardner (2017), 235.

65 SW 1/7, 338-339. 
im Text einführt, als ein etabliertes Merkmal seiner Naturphilosophie. ${ }^{66}$ Daher muss dieser Unterschied eine andere Unterstützung haben als seine Analyse des Moralbewusstseins. Mit anderen Worten gesagt unternimmt Schelling, seinen zweigleisigen Denkansatz aufrechterhaltend, den Versuch, eine Theorie der internen Dynamik des Seins zu entwerfen, die den Beschränkungen unterliegt, die aus Respekt vor der Phänomenologie des Guten und Bösen folgen.

\section{Schelling und der weiche Naturalismus}

Die Raffinesse und Komplexität von Schellings theoretischer Arbeit zum Freiheitsbegriff sollte mittlerweile offensichtlich sein. Dennoch verträte eine Mehrheit zeitgenössischer Philosophen zweifellos die Ansicht, dass der Denkansatz, dem die spekulativen Unternehmungen der Deutschen Idealisten folgten, unwiederbringlich der Vergangenheit angehört, und wir nunmehr über glaubwürdigere Denkmittel verfügen, um mit vergleichbaren Problemstellungen umzugehen. Meiner Auffassung nach kann man sich allerdings über ein Projekt wie das Schellings nicht so einfach hinwegsetzen. Um dies zu zeigen, werde ich abschließend die Freiheitsschrift mit dem klassischen Essay von Peter Strawson vergleichen, der sich gleichfalls mit der Phänomenologie und der Metaphysik der Freiheit beschäftigt. Strawsons Arbeit auf diesem Gebiet war wegweisend für den „weichen Naturalismus“, der gegenwärtig gemeinhin als die Lösung vieler Probleme gesehen wird, denen die spekulativen Bestrebungen der Idealisten galten. ${ }^{67}$

In „Freedom and Resentment“ hat Strawson bekanntermaßen geltend gemacht, dass die Übernahme der objektivierenden Einstellung, von der einige unerschütterliche Deterministen glauben, sie sei als Folgerung aus der theoretischen Wahrheit ihrer Lehre zu schließen, die Verallgemeinerung eines Standpunktes beinhielte, den wir gegenwärtig nur dann einnehmen, wenn besondere Umstände oder die ungewöhnlichen Charaktereigenschaften der betreffenden Person uns davon überzeugen, dass unsere normalen Reaktionen dem Anderen gegenüber suspendiert werden sollten. Strawsons Überzeugung nach ist dies

66 SW 1/7, 358.

67 Der weiche Naturalismus ist in der gegenwärtigen Philosophie weitverbreitet. Er findet sich nicht nur im Konstruktivismus in der Folge von John Rawls, wie etwa bei Christine Korsgaard, und bei Philosophen in der Tradition von Ludwig Wittgenstein wie John McDowell, sondern auch bei Hegel-Interpreten wie Robert Pippin und Terry Pinkard sowie bei Vertretern der Kritischen Theorie wie Jürgen Habermas und Axel Honneth. In die Diskussion eingeführt wurde der Terminus offenbar von Strawson (vgl. Strawson 1987, 13 u. ö.). 
jedoch „praktisch undenkbar“. Der Gedanke sei nicht ernst zu nehmen, dass „die allgemeine theoretische Überzeugung unsere Welt so sehr ändern könnte, dass es in ihr so etwas wie interpersonale Beziehungen, wie wir sie gemeinhin verstehen, nicht mehr gäbe“. ${ }^{68} \mathrm{Zu}$ behaupten, die Preisgabe solcher Beziehungen sei die rationale Konsequenz einer bestimmten theoretischen Wahrheit, stellte den eigentlichen Sinn des Terminus „rational“ in Frage. In welchem Sinne sollte es - auch wenn sich eine solche Wahrheit aufstellen ließe - nicht rationaler sein, die wesentlichen Eigenschaften unserer menschlichen Lebensform zu erhalten? Zugleich leugnet Strawson, dass wir, um unsere spontanen sittlichen Reaktionen von Tadel, Verbitterung, Verärgerung $u$. a. gemeinsam mit deren positiven Korrelaten zu stützen, darauf rekurrieren sollten, was er die „obskure und beunruhigende Metaphysik des Libertarismus“ nennt. ${ }^{69}$ Solche Reaktionen geben unserer menschlichen Natur Ausdruck, und jenseits dessen gebe es nichts, auf das wir uns berufen können oder müssen.

Während sowohl Schelling als auch Strawson von der existenziellen Beschreibung als einem nicht zu ersetzenden Element ihrer Strategien Gebrauch machen (Strawsons Abhandlung besteht zu großen Teilen nicht aus philosophischen Argumenten im normalen Sinne, sondern einfach aus einer Anhäufung phänomenologischer Beschreibungen), gibt es zwischen ihnen offenkundig maßgebliche Unterschiede. Obwohl Schelling nicht glaubt, dass ein deterministisches System, so solide es auch aufgebaut ist, uns dazu bringen kann, das Gefühl der eigenen Freiheit zu bestreiten, behauptet er erstens (worauf bereits verwiesen wurde), dass unsere Auseinandersetzung mit der Beziehung von Freiheit und Notwendigkeit den eigentlichen Kern der Philosophie bildet und diese belebt. Dies bedeutet, dass für Schelling die von Strawson vorgeschlagene entdramatisierende Problemlösung, in der die De-facto-Einwilligung in den Determinismus (oder zumindest eine naturalistische Abneigung gegen den Libertarismus) als vereinbar mit unseren üblichen sittlichen Praktiken des Lobens und Tadelns, des Belohnens und Strafens gilt, keine Option ist. Gewiss vermag der Determinismus unser tief verwurzeltes Freiheitsbewusstsein letztlich nicht zu überwinden, aber das Versäumnis, den „Zusammenhang des Begriffs der Freiheit mit dem Ganzen der Weltansicht“ aufzuzeigen, kann, so Schelling, den Begriff der Freiheit „wankend“ und für den wiederkehrenden skeptischen Zweifel anfällig machen. ${ }^{70}$ Für Schelling ist ferner der Konflikt zwischen der objektivierenden Perspektive auf die Menschen und der existenziellen Perspektive der ersten Person nicht einfach ein Disput im

68 Ders. (2008), 12.

69 Ebd., 27.

70 SW 1/7, 338. 
Elfenbeinturm, sondern ein beunruhigendes Merkmal moderner Gesellschaften. Der „Zwiespalt von Kopf und Herz“, auf den Schelling andernorts im Text verweist, ${ }^{71}$ lässt sich als Vorahnung der Bedrohung dadurch verstehen, was später als „Nihilismus“ bekannt wurde. ${ }^{72}$ Dies hat zur Folge, dass Schelling, im Unterschied zu Strawsons Selbstgefälligkeit, vom Erfordernis überzeugt ist, Freiheit und Notwendigkeit durch ein philosophisches Bemühen miteinander zu verbinden, das über die Beschreibung unserer eingelebten sittlichen Reaktionen oder die Berufung auf unser unbesiegbares Gefühl der Freiheit weit hinausgeht. Beide bedürfen der Versöhnung in zwei verschiedenen Richtungen. Obwohl unsere Handlungen sich möglicherweise zwangsläufig aus unserem Charakter ergeben, ist zu zeigen, dass dieser doch als in einem nichtempirischen Sinn frei gewählt verstanden werden kann. Zweitens müssen wir uns aber auch vergewissern, dass unter der Annahme einer solchermaßen grundsätzlichen Freiheit langfristig nicht Chaos entsteht: Denn die Überzeugung, dass die Welt - im Innersten - sittlich chaotisch ist, wäre möglicherweise nicht weniger nihilistisch als der Determinismus. Dies ist der Punkt, an dem Schellings Argument, die Welt müsse die Form eines Systems annehmen, ihre motivationale Bedeutung erhält und, in der Ausdrucksweise der Zeit gesprochen, zum Projekt einer Theodizee wird.

Hierauf werden einige vielleicht noch immer erwidern, dass aus der Theorieperspektive Strawsons oder der des „weichen Naturalismus“ aus gesehen dieses alles grandioses Gewese und damit unnötig sei. Der objektivierenden Perspektive auf Menschen zu gestatten, $\mathrm{zu}$ unangemessenen Gelegenheiten oder in unzumutbarer Weise in die Lebenswelt einzudringen, ist zweifellos eine Gefahr (wenn wir etwa von Politikern angehalten werden, uns so zu verhalten, als würden verschiedene „Rassen“ in einen darwinistischen Kampf um die Vorherrschaft getrieben). Solange wir aber eine gesunde Arbeitsteilung zwischen beiden Perspektiven aufrechterhalten, werde kein Schaden auftreten. Gleichzeitig könnten wir jede Art metaphysischer Überschwänglichkeit vermeiden. Wie Gardner jedoch andernorts argumentiert, ist der Vertreter des weichen Naturalismus angesichts der Gefahr, dem harten Naturalismus nachzugeben, weiterhin auf eine Meta-Perspektive verpflichtet, von der aus sowohl die Perspektive der ersten Person als auch die objektivierende Perspektive als solche gebilligt werden, die auf je eigene Weise die Realität wahrhaft enthüllen. ${ }^{73}$ Falls nämlich der Vertreter des weichen

71 Ebd., 348.

72 Für eine Interpretation der Spätphilosophie Schellings als Antwort auf die Gefahr des Nihilismus, die sich (meinem Verständnis nach) bereits in der Freiheitsschrift abzeichnet, vgl. die Diskussion bei Iber (1999), 191-210.

73 Vgl. Gardner (2007), 33-34. 
Naturalismus von der Ontologie Abstand nehmen und argumentieren sollte, dass Zweckmäßigkeit, Intentionalität, Zurechenbarkeit und dergleichen nur innerhalb der Lebenswelt real erscheinen, dann könne der Vertreter des harten Naturalismus antworten: „Genau!“ So gesehen besteht zwischen Schelling und den Vertretern des weichen Naturalismus kein Unterschied hinsichtlich der philosophischen Höhenlage (wohl aber hinsichtlich des Grades der Ausarbeitung) des Standpunkts, der die Berechtigung beider Perspektiven geltend macht. ${ }^{74}$

Letztere Behauptung wird zweifelsohne kontrovers erscheinen, sodass es vielleicht sinnvoll ist, sie etwas länger zu erwägen. In seiner Vorlesungsreihe Skeptizismus und Naturalismus hat Strawson die in „Freedom and Resentment“ vorgestellte Position weiter ausgearbeitet. Der Aufsatz hatte den starken Eindruck hinterlassen, dass die Wahrheit des Determinismus (oder zumindest die Unglaubwürdigkeit des Libertarismus) nicht angefochten wurde. Im Buch allerdings ist Strawson weniger parteiisch. Er vertritt, was er eine „bestimmte letztendlich bestehende Relativität in unserer Konzeption des Wirklichen“"75 nennt: Eine „Versöhnung anscheinend konfligierender Auffassungen“ lasse sich „dadurch erreichen, daß die Konzeption des Wirklichen - dessen, was wirklich existiert oder wirklich der Fall ist - auf verschiedene Standpunkte relativiert wird“"76. Zugleich bestreitet er, dass es jenseits des harten oder „reduktionistischen“ Naturalismus und des weichen oder „katholischen“ Naturalismus irgendeinen „höheren Standpunkt“"77 gebe. Seine Gedankenführung ist jedoch nicht schlüssig. Die vorgeschlagene Versöhnung setzt bereits einen höheren Standpunkt voraus, der die Perspektiven des harten Naturalismus und der Lebenswelt relativiert (im Widerspruch zur Auffassung ihrer Vertreter). Zudem hat dieses Manöver die „anscheinend konfligierende[n] Auffassungen“ nicht versöhnt: Diese Perspektiven gleichen nämlich nicht zwei verschiedenen Perspektiven z. B. auf denselben physischen Gegenstand, da sie miteinander unvereinbare Behauptungen hinsichtlich des Aufbaus der Welt formulieren. Falls sie wahrhafte „Konzeptionen des Wirklichen“ sein sollten, denen - statt bloße Vorstellungen zu sein - eine

74 Erwähnenswert ist, dass in Strawsons Gebrauch eine Asymmetrie zwischen hartem und weichem Naturalismus besteht. Ersterer ist „reduktionistisch“ und gewährt seinem Gegenüber eine allenfalls epiphänomenale Geltungskraft. Der weiche Naturalismus hingegen gewährt dem harten Naturalismus eine nicht ausschließliche, aber irreduzible Geltungskraft, wiewohl eine solche, die dem Selbstverständnis des harten Naturalismus widerspricht. Diese Asymmetrie findet ihren treffendsten Ausdruck in Strawsons Bezeichnung „,katholischer Naturalismus“, die er als ein Synonym für „weichen Naturalismus“ anbietet. Vgl. Strawson (1987), 13, 14, 49, 85 u. 107. 75 Ebd., 53.

76 Ebd., 106, Hervorh. getilgt.

77 Ebd., 47. 
wenngleich qualifizierte Gültigkeit zukommen soll, bliebe immer noch zu zeigen, wie die Wirklichkeit beide beherbergen kann. Strawson bietet kein Argument, um zu zeigen, dass eine solche Unternehmung unmöglich ist. Er setzt einfach voraus, dass ein Anspruch auf Ganzheitlichkeit nur auf der Überdehnung der einen oder anderen Perspektive gründen kann, und schließt aus, dass eine neue Konzeption beide versöhnen könnte. Er bleibt damit gefangen im performativen Widerspruch, die Möglichkeit einer Metaperspektive zu bestreiten. Daraus lassen sich allgemeine Lehren über die Stellung des weichen Naturalismus ziehen.

\section{Schluss}

Wie seine eigene Kritik am weichen Naturalismus erwarten lässt, bietet Gardners Abhandlung über die Freiheitsschrift eine wohlwollende, subtile und beziehungsreiche Deutung des Textes an. Seinem Interpretationsvorschlag zufolge besteht Schellings Leistung darin, die Unbegreiflichkeit - d. h. jene der Freiheit - nicht länger (wie Kant) als ein Anzeichen für die Grenzen unserer Erkenntnisvermögen zu betrachten, sondern als ein Merkmal der Wirklichkeit als solcher (dieser Schritt wird durch Schellings Behauptung unterstrichen, dass der „Grund“ die „unergreifliche Basis der Realität“ verkörpere, den „nie aufgehende[n] Rest, das, was sich mit der größten Anstrengung nicht in Verstand auflösen läßt““ ${ }^{78}$. Gardner fasst zusammen:

\footnotetext{
Daher lässt sich sagen, dass Schelling zwar die Unbegreiflichkeit der Freiheit, wie sie von Kant vertreten wurde, ausgeräumt hat, ohne jedoch zur Erkenntnis der Freiheit beizutragen. In Bezug auf das Problem des Bösen ist Schellings Leistung, dass er es ermöglicht hat, die Frage danach, warum das Böse existiere, mit der Replik „Ohne Grund“ zu beantworten - er hat die Bedingungen formuliert, unter denen diese Antwort als korrekt und vollständig gelten darf. Die Freiheitsschrift steht zwar in der Tradition der Theodizee, indem sie Gott von Schuld freispricht und uns mit dem Sein versöhnt, aber die Mittel, deren sich Schelling dabei bedient, sind denen Leibniz' diametral entgegengesetzt. ${ }^{79}$
}

Schelling glaubt jedoch nicht, dass die Aufgaben einer Theodizee einfach durch Offenlegung der bloßen Faktizität der Freiheit und des Bösen erfüllt werden können. Einerseits möchte er (im Unterschied zu Kant) angesichts der Zwangslagen menschlichen Selbstseins die Wahl des Bösen verständlich machen - obwohl sie letztlich wohl nicht rational ist, ist sie doch verständlich in der Art, wie wir

78 SW 1/7, 360.

79 Gardner (2017), 235-236. 
verstehen, warum eine Person sich möglicherweise gegen eine eigentlich lebensrettende Amputation sperrt. Außerdem möchte er begreifen, wie und warum das Böse aufhören muss - dies ist die temporale Dimension der Befriedigung der Forderung der Systematizität. Schelling hat, anders gesagt, beide Aspekte seiner dualen methodologischen Perspektive durchzuarbeiten. Dies bringt uns zurück zu der Frage, die wir am Ende unserer Überlegungen zu Gabriel offengelassen hatten: Erreicht Schelling tatsächlich sein Ziel, diese beiden Sichtweisen in Einklang zu bringen?

Die Freiheitsschrift ist in dieser Hinsicht, so würde ich behaupten, ein grandioser Fehlschlag; dies deshalb, weil Schelling noch nicht hinlänglich ausgearbeitet hat, wie der theoretische und der existenzielle Aspekt seiner Unternehmung zusammengeführt werden können; ${ }^{80}$ oder er war, genauer gesagt, der erst recht verwirrenden Aufgabe noch nicht ganz gewachsen, diese Aspekte zusammenzuführen, ohne den Konflikt von Freiheit und Notwendigkeit, den er vom intellektuellen und seelischen Standpunkt her für unentbehrlich hält, zu unterdrücken. Letztlich weist er in der Freiheitsschrift der Theorie den Primat zu, worauf sein anfänglicher Vorschlag hindeutet, dass unser unmittelbares Freiheitsbewusstsein der „letzte[n] wissenschaftliche[n] Vollendung“ bedürfe. ${ }^{81}$ Deutlich wird dieser Theoretizismus auch in der Annahme, dass die Welt - qua Prozess göttlicher Selbstoffenbarung - sich durch sittliche Vollendung charakterisieren lässt. Anders gesagt interpretiert Schelling den Begriff einer solchen Vollendung nicht eben im Sinne einer praktischen Forderung, was zugegebenermaßen die ganze Tragweite des Begriffs nicht notwendigerweise erschöpft. Das Böse ist, so zeigt Schelling, selbstzerstörerisch, weil es regressiv zu einer reinen Partikularität tendiert, die sich ontologisch selbst aufhebt. Aber seine metaphysische Voraussage, wonach unsere Freiheit nicht zum Ergebnis haben könne, dass das Böse als eine solche Tendenz wiederholt in immer neuen Formen hervorbricht, statt langfristig gegen null zu gehen, kann er nicht begründen.

Das Problem beschäftigte Schelling weiterhin intensiv; eine tragbarere Lösung konnte er aber erst erzielen, als er in seiner ersten Münchener Vorlesung der Jahre 1827/1828 begann, den Unterschied zwischen „negativer“ und „positiver“ Philosophie herauszuarbeiten. ${ }^{82}$ Negative Philosophie wird als die A-priori-Wissen-

80 In seiner bekannten Vorlesung aus Anlass des 100. Todestags von Schelling, „Schelling und die Anfänge des existentialistischen Protestes“, charakterisiert Paul Tillich Schellings Denken als Bestreben, zwischen „Essentialem“ und „Existentialem“ zu navigieren (Tillich 1955, 204). Er schließt, dass Schelling trotz der Größe seines Werkes dieses Ziel verfehlt habe.

81 SW 1/7, 336.

82 Vgl. Schelling (1998), 83-92. 
schaft begriffenen Seins vorgestellt, aber sie kann die Frage nach dem Warum des Seins nicht beantworten, die Existenz der Welt in Hinblick auf deren Ursprung und Zweckhaftigkeit nicht verständlich machen, weil das Wirken der reinen Vernunft - Schelling pflichtet Kant nun ausdrücklich bei - wohl zur Wirklichkeit des Unbedingten strebt, diese aber nicht begründen kann. Die positive Philosophie hingegen ist eine Hermeneutik der Entwicklungsgeschichte des menschlichen Bewusstseins anhand der durch die negative Philosophie generierten Kategorien. Der Übergang zwischen negativer und positiver Philosophie erfolgt in dem Moment, in dem die Vernunft „aus sich gesetzt“ wird und sich der „unvordenklichen“ Quelle öffnet, aus der die Welt und deren Geschichte stammen. Aber ist dieser Ursprung Freiheit und somit eventuell Vernunft - oder doch nur das „Blindseyende“, die rohe, zwingende Faktizität des Seins? ${ }^{83}$ Letztlich vertritt Schelling die Auffassung, dass es keine rein theoretische Antwort auf diese Frage gebe und somit der Konflikt zwischen Freiheit und Notwendigkeit auch nicht definitiv überwindbar sei. Wir können nur sagen, dass Freiheit gewesen sein wird - soweit die Menschheit praktisch und sittlich den „Hang zum Bösen“ überwindet und es ihr gelingt, vom Bestreben abzulassen, der Grund ihrer eigenen Freiheit zu werden. Von diesem Futur II abhängig bleibt die menschliche Existenz in der Schwebe.

Aus dem Englischen von Veit Friemert

\section{Literatur}

Allison, H. (1990), Kant's Theory of Freedom, Cambridge.

Beiser, F. (1987), The Fate of Reason, Cambridge, Mass.

Dews, P. (2008), The Idea of Evil, Oxford.

Dörendahl, R. (2011), Abgrund der Freiheit. Schellings Freiheitsphilosophie als Kritik des modernen Autonomie-Projekts, Würzburg.

Ferrer, D., u. Pedro, T. (2012) (Hg.), Schellings Philosophie der Freiheit, Würzburg.

Franks, P. (2005), All or Nothing. Systematicity, Transcendental Arguments, and Skepticism in German Idealism, Cambridge, Mass.

Gabriel, Markus, Das Absolute und die Welt in Schellings Freiheitsschrift, Bonn 2006.

Gardner, S. (2007), The Limits of Naturalism and the Metaphysics of German Idealism, in: Hammer, E. (Hg.), German Idealism. Contemporary Perspectives, London, 19-49.

Gardner, S. (2017), Die Metaphysik der menschlichen Freiheit. Von Kants transzendentalem Idealismus zu Schellings Freiheitsschrift, in: Deutsche Zeitschrift für Philosophie 65.1, 211-238.

83 Vgl. z. B. ders. (1977), 154-160. 
Heidegger, M. (1971), Schellings Abhandlung über das Wesen der menschlichen Freiheit, Tübingen.

Iber, C. (1999), Subjektivität, Vernunft und ihre Kritik, Frankfurt am Main.

Kant, I. (1977a), Die Religion innerhalb der Grenzen der bloßen Vernunft, in: Werkausgabe 8, hg. v. Weischedel, W., Frankfurt am Main, 649-879.

Kant, I. (1977b), Metaphysik der Sitten, in: Werkausgabe 8, hg. v. Weischedel, W., Frankfurt am Main, 309-634.

Kant, I. (1974), Kritik der reinen Vernunft (= Werkausgabe 3-4), hg. v. Weischedel, W., Frankfurt am Main.

Nagel, T. (2012), Mind and Cosmos, Oxford.

Paetzold, H., u. Schneider, H. (2010) (Hg.) Schellings Denken der Freiheit. Wolfdietrich Schmied-Kowarzik zum 70. Geburtstag, Kassel.

Roux, A. (2010) (Hg.), Schelling en 1809. La liberté pour le bien et pour le mal, Paris.

Schelling, F. W. J. (1998), System der Weltalter. Münchener Vorlesung 1827/28 in einer Nachschrift von Ernst von Lasaulx, hg. v. Peetz, S., Frankfurt am Main.

Schelling F. W. J. (1977), Philosophie der Offenbarung 1841/1842, hg. v. Frank, M., Frankfurt am Main.

Schelling F. W. J. (1870), Aus Schellings Leben in Briefen 2: 1803-1820, hg. v. Plitt, G. L., Leipzig.

Schelling F. W. J. , Sämmtliche Werke, hg. v. Schelling, K. F. A., Stuttgart u. Augsburg [SW].

Strawson, P. (2008), Freedom and Resentment [1962], in: Freedom and Resentment and Other Essays, Oxford u. New York, 1-28.

Strawson, P. (1987), Skeptizismus und Naturalismus, übers. v. Istase, M. N., u. Soskey, R., Frankfurt am Main; Orig.: Scepticism and Naturalism. Some Varieties, New York 1985.

Tillich, P. (1955), Schelling und die Anfänge des existentialistischen Protestes, in: Zeitschrift für philosophische Forschung 9.2, 197-208.

Zuerst erschienen als: Peter Dews, Theory Construction and Existential Description in Schelling's Freiheitsschrift, in: British Journal for the History of Philosophy 25.1 (2017), 157-178. Copyright $\odot$ BSHP, reprinted by permission of Taylor \& Francis Ltd, www.tandfonline.com, on behalf of BSHP. 